\title{
Characterization of turbulent latent and sensible heat flux exchange between the atmosphere and ocean in MERRA
}

J. Brent Roberts ${ }^{1}$, F. R. Robertson ${ }^{1}$, C. A. Clayson ${ }^{2}$, M. Bosilovich ${ }^{3}$

Research Supported by NASA Energy and Water Cycle Study (NEWS) 1NASA/MSFC , ${ }^{2} \mathrm{WHOI},{ }^{3} \mathrm{NASA} / \mathrm{GSFC} / \mathrm{GMAO}$ and NASA Modeling, Analysis and Prediction (MAP) Programs

Research Objectives
The recently produced Modern Era Retrospective-Analysis for Research and Applications (MERRA; Rienecker et al. 2011) provides a high-resolution dataset that can be used to examine components of the Earth's surface energy and water balance. Latent and sensible heat exchanges between the ocean and atmosphere are fundamental components of these balances and are the focus of this study. The primary objectives are to characterize the MERRA surface energy fluxes with respect to

1. Accuracy against direct measurements;

2. Large scale spatio-temporal variability and representation of extremes;

3. Connection to forcing by the data assimilation system.

\section{Summary Points}

MERRA produces estimates of the turbulent fluxes that agree very well with observational estimates for average conditions; however, it is distinct in amplitude with a particularly weak representation of the surface heat fuxus over the western boundary currents and in conditions of very weak and very strong near-surface stratification. A weaker covariability between wind speed and temperature/moisture stratification than observed exists.

MERRA has slightly weaker seasonal variability of the latent and sensible heat fluxes compared to an observational ensemble estimate. It tends to under-represents the occurrence of strong, episodic events compared with observations in the Northern Hemisphere mid-latitudes.

Data assimilation, as expected, tends to drive the analysis closer to the observational ensemble; the impact on near-surface variables contains systematic response to the changing observing system and could introduce artificial trends into the analysis.

\begin{tabular}{|l|} 
Observational Database \\
High-quality, direct in situ measurements of the turbulent latent and \\
sensible heat fluxes and near-surface variables serve as a standard \\
against which the veracity of turbulent flux productucts are comparend. The \\
SEAFLUX (Curry et al. 2004) program has compiled a arge datases of \\
these measurements and are utilized in this study for validation \\
purposes. The spatial and temporal distribution of these observations \\
are characterized below (Fig. 1).
\end{tabular}

Figure 1.

\section{In}

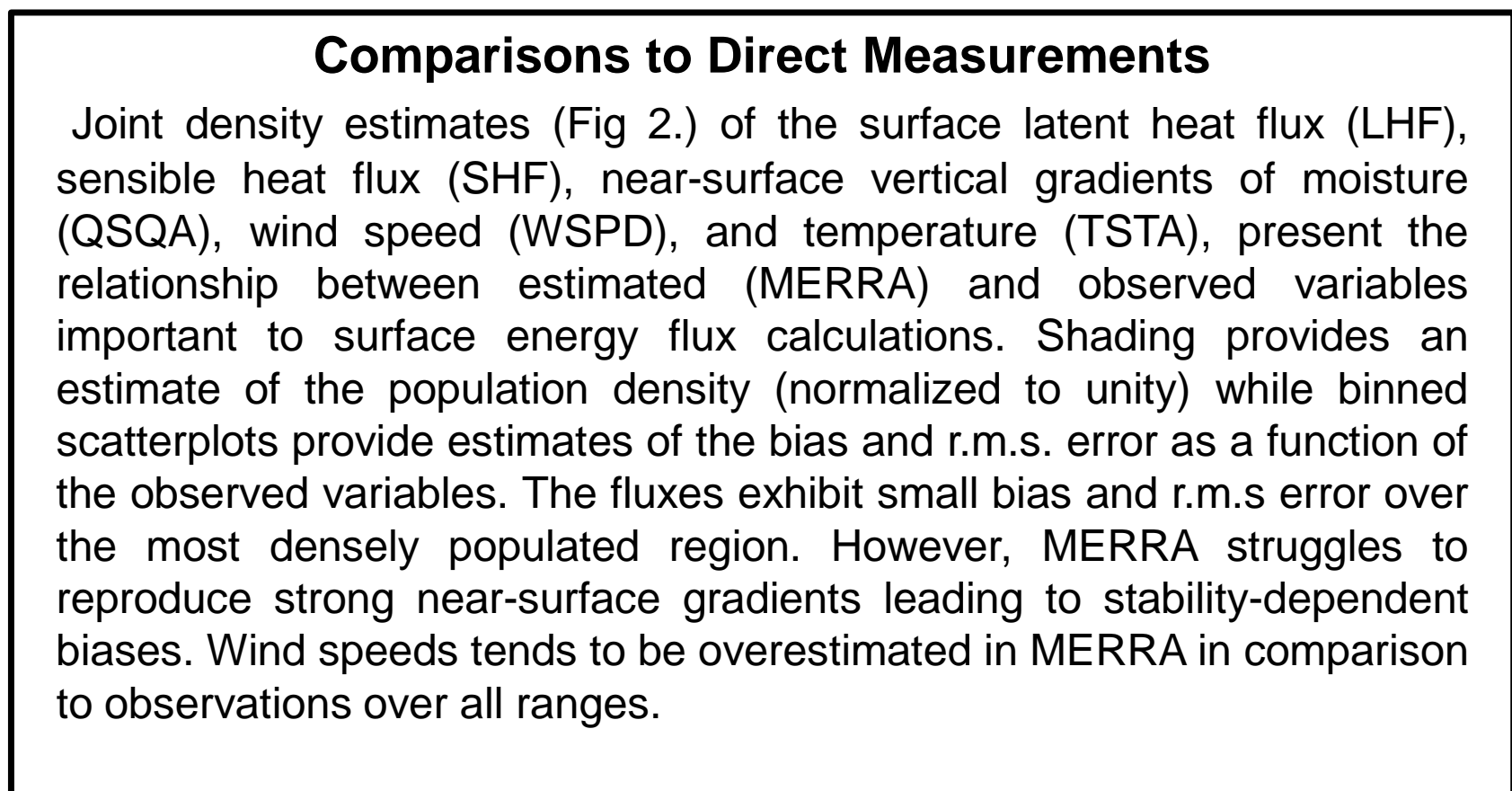

Figure 2.

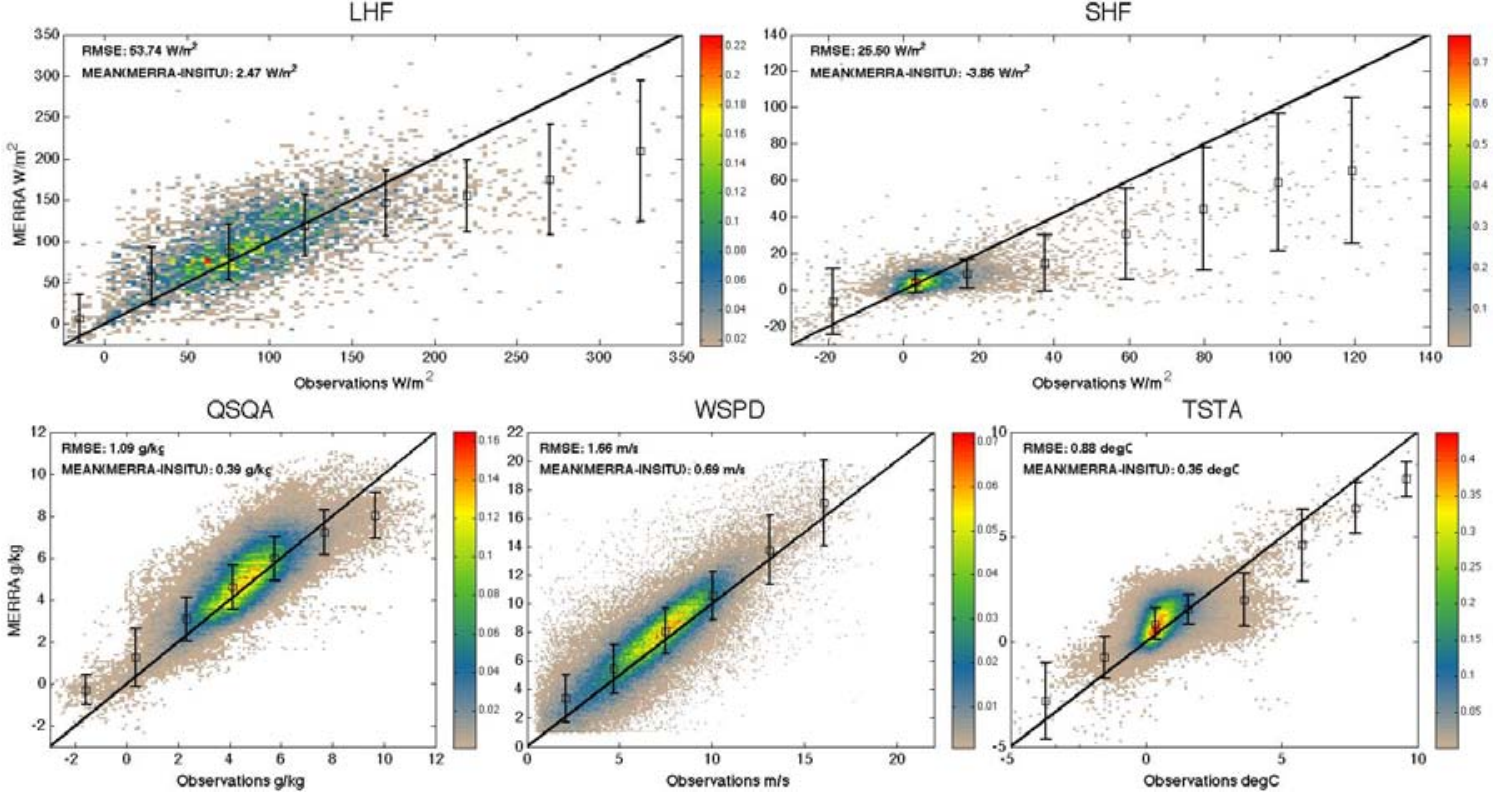

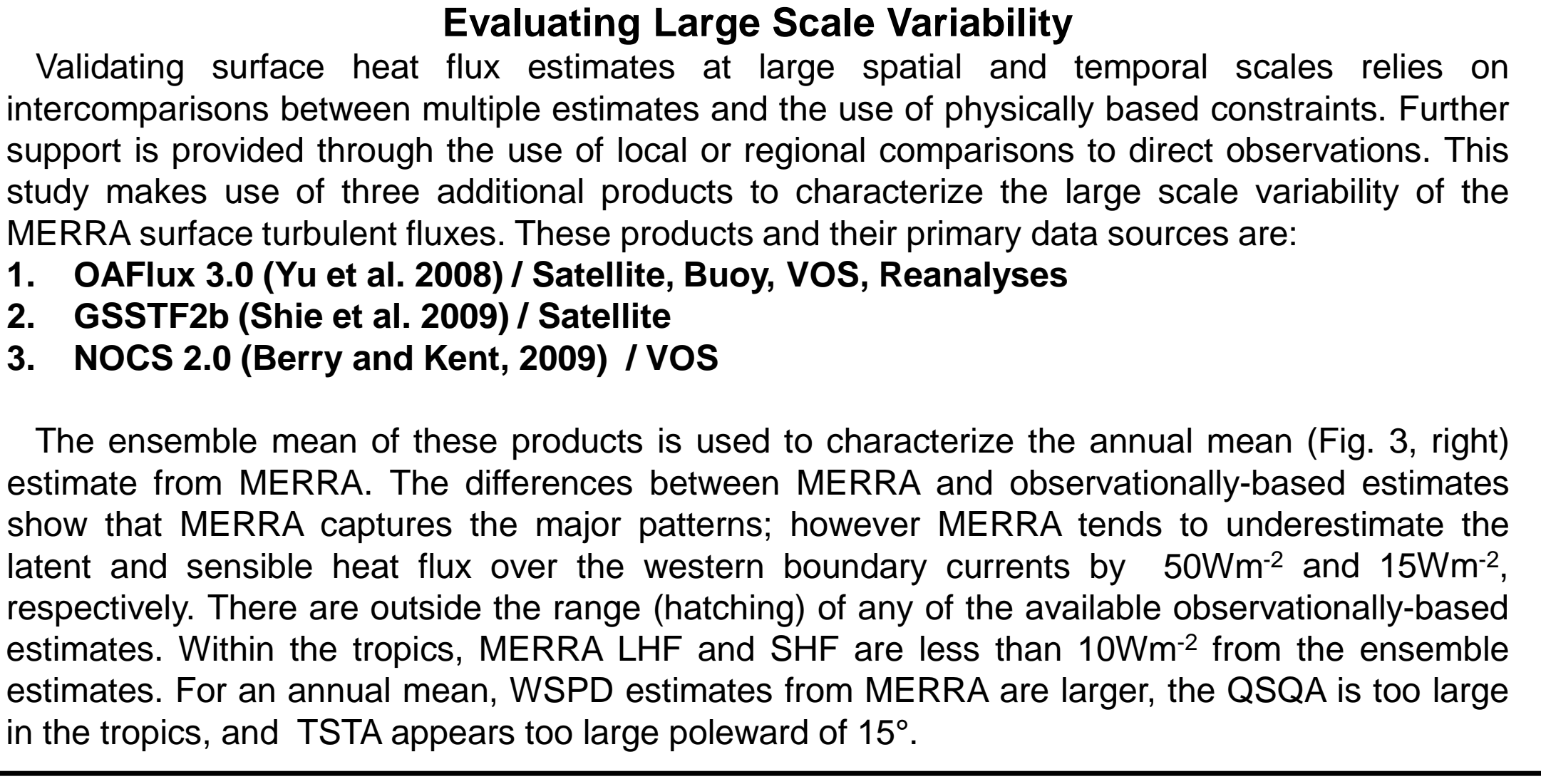
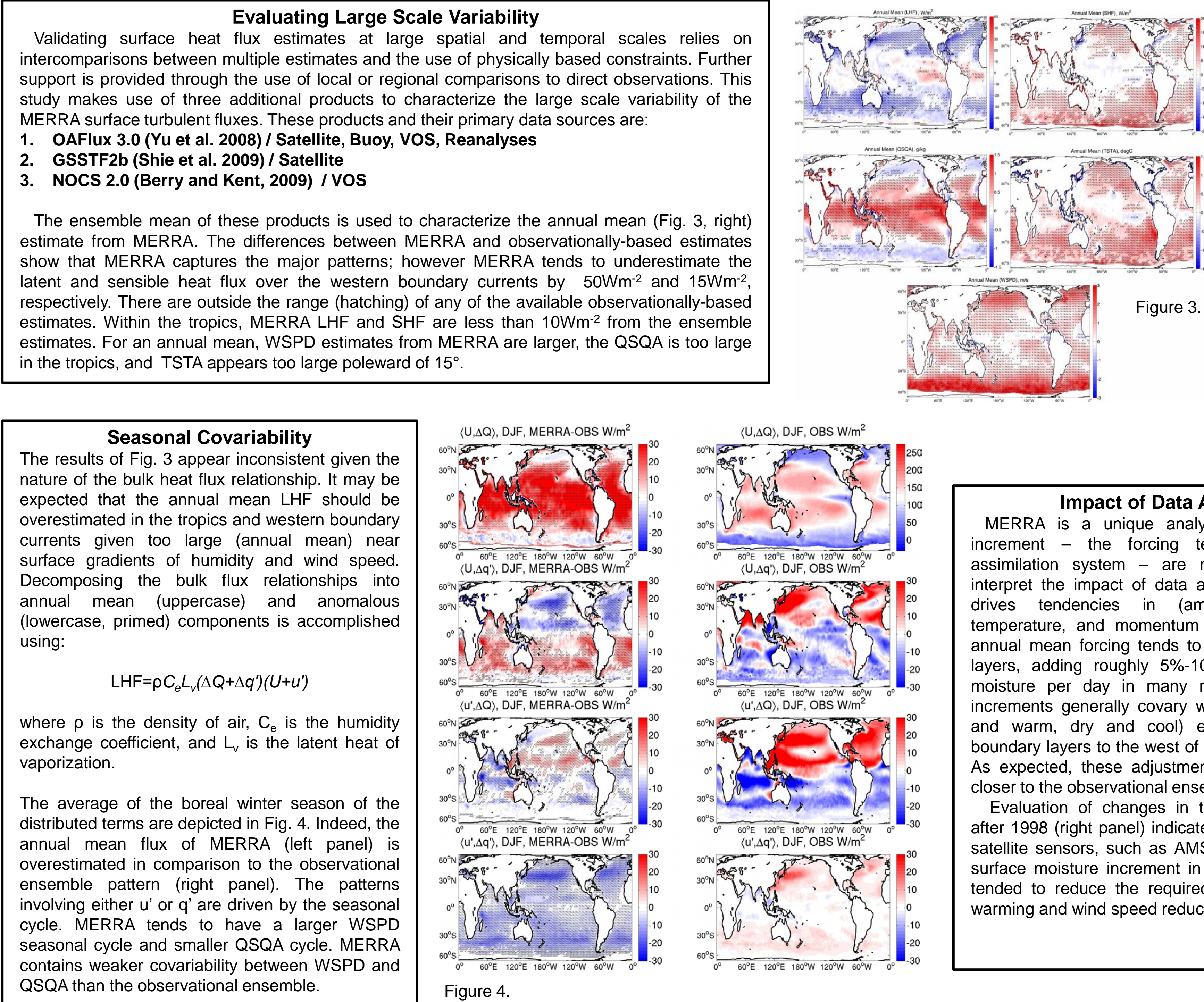
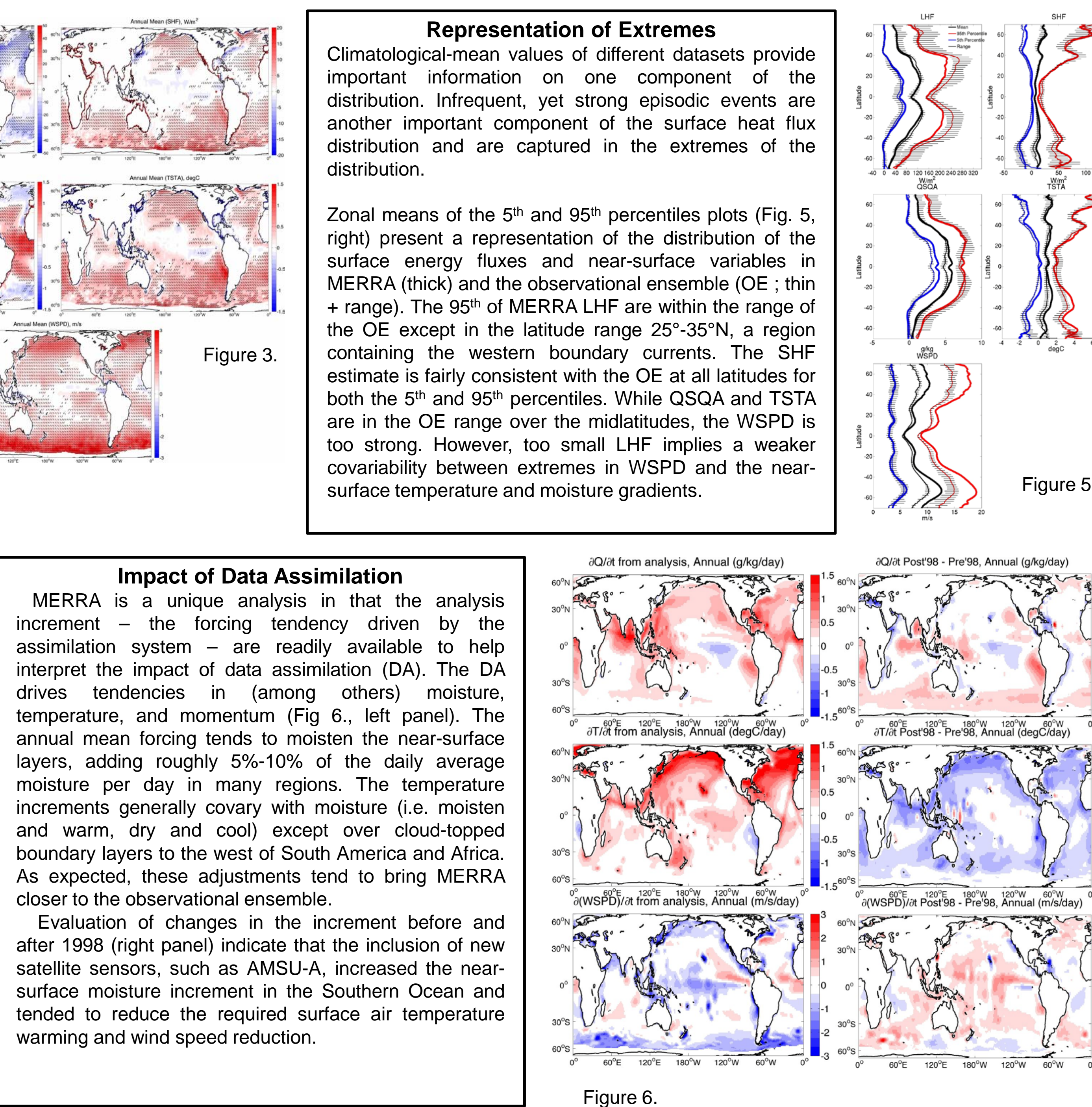

Figure 6. 